\title{
EDUCACIÓN AMBIENTAL Y PARTICIPACIÓN EN COLOMBIA Y BRASIL
}

\author{
Luisa Fernanda Mejia Toro ${ }^{1}$ \\ Marcos Sorrentino ${ }^{2}$
}

\begin{abstract}
Resumen: Brasil y Colombia son países líderes en biodiversidad, riqueza natural y cultural, y al mismo tiempo presentan conflictos internos en los que la violencia pone en jaque la viabilidad de estos sistemas. La histórica violencia que ha manchado sus territorios ha generado estructuras de poder en los que la democracia se ha visto gravemente afectada, debilitando las formas de participación política y social de sus comunidades. En este texto reflexionamos como la educación ambiental, tal como es propuesta en estos países, tiene el potencial de ser una estrategia formadora y política. El dialogo, el reconocimiento y empoderamiento del territorio son pilares defendidos por la educación ambiental latinoamericana; pues con ellos suscita escenarios de participación en los que la población puede movilizar las transformaciones culturales, sociales y naturales que necesitan en estos países.
\end{abstract}

Palabras clave: Educación Ambiental; Violencia; Participación Comunitaria; Democracia.

Resumo: Brasil e Colômbia são países líderes em biodiversidade, riqueza natural e cultural, e ao mesmo tempo apresentam conflitos internos em que a violência compromete a viabilidade desses sistemas. A violência histórica que maculou seus territórios gerou estruturas de poder nas quais a democracia foi seriamente afetada, fragilizando as formas de participação política e social de suas comunidades. Neste texto, refletimos sobre como a educação ambiental, proposta nesses países, tem potencial para ser uma estratégia formativa e política. O diálogo, o reconhecimento e o empoderamento do território são pilares defendidos pela educação ambiental latinoamericana. E, por isso, promove cenários de participação em que a população possa mobilizar as transformações culturais, sociais e naturais de que necessita nesses países.

Palavras-chave: Educação Ambiental; Violência; Participação da Comunidade; Democracia.

1 Graduada en Licenciatura em Biología Universidad Distrital Francisco José de Caldas, Doctora en Educación Universidad Federal de Minas Gerais Brasil. e-mail: profesoraluisamejia@gmail.com. http://lattes.cnpq.br/2977733727229566.

2 Graduado en Pedagogia e Biologia, Doctor em Educación. Docente, Universidade de São Paulo. Piracicaba, SP. Brasil. email: sorrentino.ea@gmail.com.br. http://lattes.cnpq.br/1555965032742989. 
Abstract: Brazil and Colombia are leaders in biodiversity, natural wealth, and cultural diversity, and at the same time have internal conflicts in which violence threatens the viability of these systems. The historical violence that has stained their territories has generated power structures in which democracy has been seriously affected, weakening the forms of political and social participation of their communities. In this text we reflect on how environmental education as proposed in these countries has the potential to be a formative and political strategy. Dialogue, acknowledgement, and empowerment of the territory are pillars defended by Latin American environmental education. And therefore, it promotes scenarios of participation in which the population can mobilize the cultural, social, and natural transformations that are needed in these countries.

Keywords: Environmental Education; Violence; Community Participation; Democracy.

\section{Introducción}

¿Cómo se hace este proceso de formación de personas que transformen el mundo? Paulo Freire (1970), dice que la educación no transforma el mundo: la educación transforma a las personas que transforman el mundo. Este es nuestro desafío. ¿Cómo construir procesos educadores que potencialicen los cambios locales y globales? En este texto se defiende que la educación ambiental, desde los principios que la constituyen y los valores que propone, es una herramienta clave para movilizar los cambios urgentes y necesarios que los países necesitan. En Colombia y Brasil las propuestas nacionales y departamentales de educación ambiental EA pueden ser una puerta de entrada para la creación de espacios reales de diálogo en los que se concretice la participación, se promueva el empoderamiento y comiencen los procesos movilizadores.

Para ello lo primero es entender que lo ambiental va mucho más allá de lo natural; al referirnos a este concepto debemos reconocer la complejidad de relaciones que se establecen entre lo natural, lo social y lo cultural. $Y$ por consecuencia comprender de forma crítica el origen de estas relaciones para desde allí comenzar las transformaciones necesarias, que instauren sociedades basadas en la comprensión y cuidado de la diversidad, el dialogo y el respeto mutuo. En segundo lugar, debemos reconocer que la EA presenta una estructura dinámica, flexible que supera las fronteras establecidas entre la educación formal y la no formal. En este sentido promueve la participación de la comunidad en el proceso educativo, pues la vinculación de ésta con la escuela constituye una condición indispensable para poner al sujeto que aprende en contacto con el medio del cual hace parte, posibilitando una interacción permanente entre ambos (ALVINO; SESSANO, 2008). Es en estos cimientos que se instaura la EA latinoamericana, compromisos que dialogan con las necesidades que hoy deben trabajarse con el pueblo brasileño y colombiano para que puedan continuar luchando por la garantía de los derechos, los territorios y con esto construir territorios de paz.

En América Latina, la EA se materializa, desde los años 70, el desafío de la formación ambientalista surge en medio de la convergencia de las 
demandas por los derechos humanos fundamentales, por las libertades democráticas, y por la conservación, recuperación y mejora del medio ambiente y de las condiciones de vida. Proyectos, programas y políticas públicas destacan la necesidad de una educación permanente, continua, articulada y con la totalidad de los sujetos; aun así, no han sido suficientes para detener el avance de la degradación ambiental. Después de la segunda guerra mundial y, especialmente, después de las primeras percepciones de los impactos y consecuencias de la llamada revolución verde y de la comprensión de las demandas y propuestas denominadas contraculturales de los jóvenes a finales de los años 60's, quedaron claros los males del modo hegemónico de producción y consumo presente en las sociedades capitalistas y, en las dichas socialistas. Empieza a forjarse un conjunto de ideas e ideales nombrados por y a partir de distintos autores, como nuevo movimiento social, movimiento alternativo, ecológico o ambientalista (SORRENTINO et al., 2017).

El quehacer educador ambientalista latinoamericano sabe que es esencial ir y estar con el pueblo y por esto busca aprender con la historia, con tradición, y con los métodos y técnicas de la educación popular, presente en todo el continente; promoviendo la convergencia entre lo social y lo ambiental, de donde emergen prácticas políticas y pedagógicas diversas. Teniendo en cuenta lo anterior, es necesario pensar en procesos pedagógicos de EA que promuevan la inclusión de cada uno a partir de su perspectiva, de su particular forma de ver y estar en el Planeta. No hay proceso educador que se sustente si es puntual. EA significa cambios culturales, y estos, a su vez, exigen que la EA sea permanente, continuada, articulada y con la totalidad. Esa frase debe ser adoptada como un mantra de la EA que necesita ser incluido en todos los procesos que se propongan los educadores y ambientalistas - permanente, continuada, articulada y con la totalidad.

\section{Educación Ambiental y Participación}

Existen dos principios para la construcción de estados-nación en busca de la felicidad de los humanos: el primero, es la regulación y el segundo es la emancipación. Boaventura de Sousa Santos (2012) opina que ambos necesitan ser fundamentos de las políticas públicas. Estos principios serían la base de la perspectiva de la formación de personas comprometidas con transformaciones socioambientales. El principio de la regulación ha sido ampliamente desarrollado en las últimas décadas en América Latina- normas legales, saneamiento ecológico-económico del uso de la tierra, licenciamiento, fiscalización, pagos por servicios ambientales, certificación, son algunos ejemplos de instrumentos de políticas públicas utilizados por el estado y por la sociedad. Pero, el principio de la emancipación ha sido nublado, obscurecido, marginalizado por una educación ambiental prescriptiva y normalizadora. Hay que rescatar los fundamentos y utopías educadoras y ambientalistas para alimentar una EA comprometida con la emancipación crítica de los humanos y de sus sociedades. 
Las convergencias entre lo social y lo ambiental, entre el encantamiento con la vida en toda su diversidad y la lucha por la reforma agraria, la alfabetización y las otras formas de justicia social, entre lo emocional y lo racional y tantos otros encuentros dialógicos entre opuestos (aparentes o reales), ha marcado profundamente la EA latinoamericana y la ampliación de su capacidad de incidencia en la arena pública dependerá de un balance sobre sus impases y dificultades. Y es el esfuerzo por conseguir el bienestar de estas convergencias, lo que puede garantizar el inicio de una verdadera situación de paz en un país.

El aporte que se hace desde la EA es pensar un tipo de educación diferente que fortalezca y apoye los procesos de empoderamiento de las comunidades, paso fundamental en la construcción de este proyecto de transformación de sociedad. En este contexto, se espera que la educación ayude a sentar las bases para la construcción de un nuevo tipo de cultura, de una nueva forma de saber y entender, que cuestione y critique el sistema hegemónico de poder para la construcción de una nueva sociedad. Es este potencial el que permite creer que la EA puede mediar en procesos de identificación y reconstrucción de territorios afectados por los conflictos y en el establecimiento de sociedades nuevas que lideren las luchas en el que el cuidado del territorio sea el protagonista.

Consolidar una idea en el que sea el ciudadano el actor principal de la transformación y que sea en comunidad como se construyan nuevos modelos de valor y parámetros de actuación; repensar ese desafío epistemológico, puede ayudarnos a imaginar la reconstrucción de una visión global, multidimensional e interdisciplinar, que asocie la naturaleza y la cultura como eje fundamental para comprender mejor el mundo en el cual vivimos, y así, permitirnos imaginar un proyecto social y político que sea capaz de asumir los desafíos impuestos por el contexto actual de la globalización (MARíN, 2009).

\section{La Participación en el Contexto Brasileño}

En Brasil, se vive una realidad en constante transformación y lucha; la colonización, la esclavitud y la explotación de los recursos, han modificado las relaciones sociales, las relaciones con la naturaleza, y con el territorio:

La colonización del territorio brasileño ha tenido varios impulsos de avance de la frontera, alimentados por diferentes ciclos de extracción y producción. Esos ciclos fueron, en general poco relacionados unos con los otros, o a veces sin ninguna conexión. Se difundieron por diferentes partes del territorio y explotaron diferentes recursos: captura de poblaciones indígenas para esclavitud; extracción de Pau-Brasil, animales exóticos y productos de la selva; cultivo de caña de azúcar, algodón y café; ganadería; minería de oro y piedras preciosas; además de otro ciclo corto como caucho y cacao. Esos pulsos causaron "saltos" sobre productos o áreas específicas dando forma al territorio (FRANCO; DRUMMOND, 2012, traducción propria). 
El proceso colonial hoy se perpetua por diferentes formas de colonialidad en el que se establece la hegemonía de saberes, de formas de ser y de poder que han instaurado la supremacía de unos grupos sociales sobre otros. Las formas de exclusión y dominio han sectorizado las necesidades, la pobreza y han encontrado en la violencia una herramienta de control político y social. Las cifras demuestran como la violencia afecta la población de modo desigual, con riesgos diferenciales que responden a género, raza, color, edad y espacio social (SOUZA; LIMA, 2007). Chesnais Jean Claude (1999) narra como la violencia urbana representada por los altos índices de muertes violentas, por ejemplo, se ha naturalizado en los medios de comunicación, pero también en el día a día de muchas comunidades, reproduciendo lo que el autor denomina una especie de "apartheid social" en el que el sentimiento de inseguridad ha promovido un malestar colectivo que se traduce en una falta de confianza en las instituciones públicas.

Este descontento acompañando de la vulnerabilidad de la joven democracia brasileña contrasta con las formas en que los grupos sociales en el Brasil se han organizado para la lucha contra estos escenarios. La historia política moderna del Brasil ha pasado por diferentes momentos para conquistar la tan anhelada democracia. Braga Sousa Maria e Acuña Chaverri Iván (2015) describen algunas fases de este proceso, que incluyen periodos de fortalecimiento del modelo de producción con Getulio Vargas, la primavera democrática donde se fomentó la participación de partidos políticos, el periodo de autoritarismo y violencia generada por la dictadura militar desde 1964 hasta la década de 1980; y el renacimiento de la democracia representado por la formulación de la Constitución política de 1988.

La experiencia de la dictadura militar provocó, en el Brasil, procesos educativos movidos por la indignación, la necesidad de cambio, la utopía y el sueño de una sociedad sustentable (FERRARO; SORRENTINO, 2011). Durante casi tres décadas después de la promulgación de la constitución, más específicamente a partir de 2003, el país vivió un periodo de desarrollo económico, inclusión social y estabilidad de las instituciones democráticas, con una fuerte presencia, aunque marginal, de la agenda ambiental en las distintas esferas del gobierno representada en el incremento de la legislación y de los aparatos estatales, así como de las instancias y formas de participación de la sociedad (MEJIA TORO, 2018). Al mismo tiempo en que se daba una notable evolución en las políticas de superación del hambre, la miseria y del apoyo a la agricultura familiar, como otros programas de inclusión social.

Infelizmente este progreso no fue suficiente para erradicar una cultura aún marcada por la esclavitud, la colonización y subordinación a los grandes intereses financieros. Desde 2016, el país enfrenta tensiones en su sistema democrático en su aparato institucional y legal impactando la educación ambiental, la conservación y recuperación de medio ambiente. Con esto, nuevamente se revela el perfil colonizado del país, (re)emergentes sectores comprometidos con la mejora de la economía, fundamentada en la exportación de bienes naturales (agrícolas, minerales y de la propia fuerza de trabajo 
humano) a bajo costo, para y por las antiguas y siempre actuantes fuerzas colonizadoras, hoy especialmente representadas por el sistema financiero internacional. Se busca disminuir el tamaño del estado brasileño por medio de cortes de recursos destinados a la educación, ciencia y tecnología, cultura, seguridad social, demarcación de tierras de las comunidades tradicionales y de la reforma agraria y medio ambiente. Se busca alimentar la economía, por medio de la disminución de derechos sociales y de los trabajadores y de la venta de empresas estatales y de la entrega facilitada de tierras y bienes ambientales y materiales para grandes conglomerados internacionales. Con la Educación ambiental sucede lo mismo, eliminando estructuras gubernamentales responsables por la implementación de una Política Nacional en el área. En el centro de esas "reformas" que siquiera servirían para alimentar la economía, un discurso contrario a la participación social y el diálogo, que defiende el uso de las armas y de la fuerza para la solución de problemas sociales, discriminando y desacreditando la diversidad, los movimientos sociales y la oposición.

El Atlas de la Violencia en el Brasil de 2019 apunto 65.602 homicidios en 2017, promovidos por el aumento de la violencia letal contra públicos específicos, entre ellos negros, población LGTBI+, jóvenes y mujeres (INSTITUTO DE PESQUISA ECONOMICA APLICADA; FÓRUM BRASILEIRO DE SEGURANÇA PÚBLICA, 2019). El Brasil está entre los países más letales para activistas y defensores de la tierra y del medio ambiente, según la ONG Global Witnes en 2019 fueron 24 asesinatos de ambientalistas, y la gran mayoría en la Amazonia siendo, 10 de las víctimas, indígenas (WELLE, 2020). Estos contextos, así como las características de la cultura política brasileña influenciaron las decisiones políticas que se tomaron en la implementación de la EA. El mito de la no-violencia brasileña, del pueblo organizado y pacífico, por una desviación de lo real o por la adjudicación de las violencias a lo excluido, ha permitido que la violencia que estructura y organiza las relaciones sociales sea naturalizada negando que el estado y la sociedad brasileña sean autoritarios.

Por su parte, el movimiento ambiental brasileño empieza con actividades y normas de protección a la naturaleza en el siglo XIX e inicio el siglo XX. Pasa por iniciativas de grupos conservacionistas, en la sociedad civil, y sobresale en la defensa del territorio, durante el periodo de la dictadura militar. En campañas de movilización popular, contra la polución y la deforestación, contra a construcción del aeropuerto metropolitano de San Pablo en área de la alta biodiversidad conservación de manantiales, entre otros, inspirando movimientos de convergencia entre luchas en defensa de la naturaleza y por libertades democráticas. Luca Andrade (2013) resalta como la capacidad de organización del pueblo brasileño es una característica fundamental en el crecimiento del movimiento ambiental. La protección ambiental empieza con tendencias hacia el cuidado de la naturaleza, donde incluso ya se hablaba de la relación Educación- protección de la naturaleza (FRANCO; DRUMMOND, 2009); hasta la defensa del territorio por parte de los brasileños, inspirando 
movimientos que se levantarían años después (SOTERO, 2008). Schittini Gilberto et al. (2012) describen tres generaciones del movimiento ambiental brasileño, que incluso se sobreponen:

La generación de los años 1920-1940, que procuraban relacionar la protección de la nacionalidad y de la identidad nacional brasileña; b. la generación de los años 1950-1980, vinculada principalmente a la fundación brasileña para la conservación de la naturaleza (FBCN), que desarrolló estrategias de creación de áreas protegidas del ecosistema; c. la generación que surgió en los años de 1990 enfocada a la conservación de la biodiversidad a partir del conocimiento científico generado por campos como la biología de la conservación y a la necesidad de negociar límites al crecimiento económico desenfrenado (ibíd., 333 traducción propia).

La diversidad de grupos sociales que se incorporaron a las luchas socioambientales generó una confianza que se tradujo en organización e incidencia sobre el poder público. A final de los años 80 e inicio de la década de los 90 del siglo pasado, en el proceso preparatorio, durante y después Rio 92, se fortalece la convergencia entre diversos actores y se forja un campo conceptual y activista nombrado como socio ambiental. Diferentes movimientos sociales asumen banderas típicas del ambientalismo: El movimiento de Trabajadores Sin Tierra (MST), por ejemplo, aprueba en sus congresos la definición de reforma agraria agroecológica; algunos partidos políticos asumen compromisos socio ambientalistas; y el consejo Nacional de Siringueros tiene en Chico Mendes uno de los exponentes de la lucha por el derecho al uso de la tierra y de los bosques y la conservación de estas.

En esta misma línea la reacción de la sociedad a las diferentes fases históricas del Brasil ha encontrado en los encuentros académicos, sociales y políticos momentos de formación política y ciudadana que teniendo la defensa por la diversidad y la democracia como intereses principales, intentan retomar un discurso que posibilite la generación de nuevas formas de participación y acción política. Por ejemplo, la realización del Acto Público "Resistir es Preciso", en 11/09/2019, en el simbólico espacio de manifestaciones en contra de la dictadura militar, en los años 70 y 80 , del Teatro de la Universidad Católica (TUCA), en Sao Paulo, abarrotándolo para escuchar el discurso de los representantes de una diversidad de movimientos sociales, religiosos, artísticos y culturales, pautados por la unión en defensa de la vida digna, de la democracia, del medio ambiente y de los derechos sociales ${ }^{3}$. Otro ejemplo es la convocatoria de marzo de 2020 del Foro Popular de la Naturaleza, protagonizado por organizaciones sindicales, movimientos de lucha por la tierra y ambientalistas.

3 Para ampliar ver <http://tvbrasil.ebc.com.br/resistir-e-preciso/episodio/como-tudocomecou\#media-youtube-1>. 


\section{La Participación en el Contexto Colombiano}

Colombia ha sido un país afectado por diferentes conflictos a lo largo de su historia que sin duda han dejado marcas culturales, físicas e históricas en todo su territorio. Luchas por la tierra, por la dominación, por los recursos, disputas políticas y partidarias entre otros. La estructura colonial genero exclusión, y con ella, silencio cualquier tipo de participación política y económica de los grupos llamados minoritarios. Colombia a pesar de ser un país capitalista, hasta hace muy poco mantenía condiciones de un sistema feudal, con terratenientes ricos y campesinos que dependían de ellos (ARISTIZÁBAL, 1999). Además del conflicto armado entre gobierno, guerrillas y grupos al margen de la ley como los paramilitares, que hasta hoy azotan el país. Un conflicto con un origen difuso para algunos, callado por otros, y del cual incluso algunos colombianos entienden poco, pero del que, aun así, se ha podido resaltar algunos detonantes: "la desigualdad social, la ausencia estatal en vastas regiones del país y por supuesto la inequitativa distribución de la tierra y su acceso a ella" (ÁNGEL, 2015 p.16).

La guerra y la corrupción han hecho del territorio una de sus grandes víctimas afectado el desarrollo ambiental del mismo; el poco respeto por lo natural, prácticas sociales y culturales conflictivas, la lucha económica y el propio conflicto armado entre otras se ven reflejadas en el uso y la relación con la tierra. Morales Lorenzo (2017) y Garavito Cesar et al. (2017) en sus trabajos describen los principales efectos ambientales de la Guerra en el territorio colombiano:

1. Un estado débil principalmente en las zonas de conflicto. La incapacidad misma del estado o el control territorial por parte de grupos armados, ha impedido la provisión y garantía de servicios básicos como educación, salud, saneamiento, vías y justicia en muchos sectores del país.

2. Una ocupación espontánea y sin planeación y el desplazamiento masivo ha generado cinturones de pobreza y miseria, así como ha empujado la frontera agrícola en las principales ciudades. Y al mismo tiempo la concentración de la tierra en manos del $13 \%$ de propietarios, algunas de ellas de manera legal, en ocasiones por la falta de opciones productivas en áreas rurales y, en otras, por el desplazamiento forzado por parte de actores armados.

3. La degradación ambiental causada por la deforestación, la pérdida de biodiversidad, la contaminación del suelo y el aumento de emisiones de gases de efecto invernadero. Todo esto asociado a diferentes factores entre ellos el desplazamiento, la erradicación de los cultivos de coca y la minería criminal; la alianza entre actores armados y terratenientes para garantizar el uso de la tierra en actividades legales e ilegales tales como cultivo de coca, ganadería, monocultivos, minería. El derrame de sustancias toxicas productos de estas actividades en suelos y fuentes de agua, o 
incluso el derrame de crudo en ataques contra la infraestructura petrolera, especialmente oleoductos; la fumigación con glifosato, o el vertimiento de mercurio por cuenta de la minería ilegal.

4. Controversialmente la presencia de grupos al margen de la ley en los territorios permitió la conservación de vastos territorios que permanecieron vedados para el estado y al mismo tiempo para proyectos industriales o de infraestructura. Este proceso, según explican los autores podía ser hecho de forma espontánea, en los casos de imposibilidad de acceso a lugares controlados por los grupos armados; pero también de manera deliberada pues en busca del control político y la regulación de la vida social y los ciclos económicos, estos grupos establecían reglamentos y restricciones a la deforestación, a la caza, la pesca, desvió de fuentes de agua entre otros; que ayudaron a proteger el medio ambiente.

El Centro Nacional de Memoria Histórica4, explica que el conflicto armado en Colombia ha generado una fragmentación entre los ciudadanos que ha influido muchísimo en la cultura y el actuar de los mismos frente a su historia y su país:

Una fragmentación del país que ha alimentado la pasividad, cuando no la indiferencia, de muchos colombianos... hay quienes se anclan en memorias sin futuro, aquellas que toman la forma extrema de la venganza, que en un escenario de odios colectivos acumulados equivale a negar la controversia y la posibilidad de coexistir con el adversario. Significan la negación radical de la democracia (p.18).

Este se convierte entonces en uno de los grandes focos a los que se deben dirigir los esfuerzos de todos cuando se piensa en la transformación del país y la construcción de la paz que tanto soñamos. El proyecto de una Colombia en paz, emprendido por diferentes gobiernos como el acuerdo de paz con la guerrilla de las Fuerzas Armadas Revolucionarias de Colombia FARC, firmado en 2016, solo se concretizará con verdaderas transformaciones sociales y culturales que implican en todo el pueblo colombiano, una forma diferente de ver, entender y construir país. Estos procesos de pacificación de grupo armados son solo uno de los pasos para la construcción de la tan anhelada paz estable y duradera que se promulga desde el gobierno colombiano.

En este contexto, trabajar con y para la gente nunca ha sido tan necesario como ahora; se hace urgente la creación de políticas y programas

\footnotetext{
${ }^{4}$ Entidad pública conformada en Colombia con el objetivo de reunir y recuperar información que permita enriquecer el conocimiento de la historia política y social de Colombia. $<$ Http://www.centrodememoriahistorica.gov.co/> 
que garanticen los derechos y el cuidado y protección de esta nación. Pues la paz se construye con todos y en un contexto de equidad y justicia, si esto no se garante, o cuando menos, se empieza a trabajar con la población, estos esfuerzos pueden ser perdidos y desencadenar conflictos mayores. Varios intentos frustrados, inconclusos o con finales trágicos, como el exterminio de la Unión Patriótica ${ }^{5}$, o el surgimiento de nuevos grupos paramilitares, algunos ahora llamados como bandas criminales Bacrim, o otros grupos al margen de la ley; así como las disidencias de la antigua guerrilla de las FARC e incluso la amenaza de conformación de una nueva guerrilla en cabeza de algunos de sus antiguos militantes; y la falta de un estado presente en todos los territorios del país; generan gran desconfianza en la población sobre la posibilidad de establecer realmente condiciones de paz y el fin del conflicto armado. Aún más cuando las necesidades básicas de todos los colombianos no están siendo suplidas, y cuando el territorio continúa en disputa.

En el caso específico del proceso de paz con las FARC, a pesar de que los diálogos públicos tomaron 4 años, solo en el momento de las campañas para el plebiscito se empezó a compartir con la población las decisiones allí tomadas. Aunque el gobierno de la época propuso desde 2014 algunas estrategias pedagógicas para integrar un dialogo pacificador en las diferentes esferas de la nación, como foros, eventos académicos y políticos, e incluso la creación de la Ley 1732 (2014) por la cual se instituye la cátedra de la paz, que proponía la entrada de este tema de forma concreta a los espacios educativos. Estos procesos fueron lentos y poco conocidos por la población, además de limitarse a espacios informativos y no de dialogo. A pesar de resaltarse dentro del mismo acuerdo, la importancia de la participación. En el acuerdo firmado por las partes, el punto dos tiene como objetivo garantizar condiciones y estrategias para fortalecer y resguardar la participación de toda la comunidad y en los demás puntos pactados se establecen diferentes órganos sociales como evaluadores, mediadores y garantes de lo pactado, enfatizando que la participación de todos y todas será la garantía máxima para el cumplimiento de las metas allí propuestas:

La participación y el diálogo entre los diferentes sectores de la sociedad contribuyen a la construcción de confianza y a la promoción de una cultura de tolerancia, respeto y convivencia en general, que es un objetivo de todos los acuerdos. Décadas de conflicto han abierto brechas de desconfianza al interior de la sociedad, en especial en los territorios más afectados por el conflicto. Para romper esas barreras se requiere abrir espacios para la participación ciudadana más variada y espacios que promuevan el reconocimiento de las víctimas, el reconocimiento y establecimiento de responsabilidades, y en general,

\footnotetext{
${ }^{5}$ La Unión Patriótica es un partido político que surgió (1985-2002) durante los diálogos entre las FARC y el gobierno de Belisario Betancur, cuyos miembros fueron exterminados sistemáticamente por extremistas de derecha.
}

revista brasileira educação ambiental 
el reconocimiento por parte de toda la sociedad de lo ocurrido y de la necesidad de aprovechar la oportunidad de la paz (PRESIDENCIA DE LA REPUBLICA; FARC-EP, 2016, p.6-7).

A pesar de estas propuestas, este proceso careció desde sus inicios de un dialogo directo con la comunidad ${ }^{6}$ pues la participación del pueblo colombiano se limitó a la de ser observadores, con excepción de pequeños espacios de presencia de sociedades de víctimas del conflicto. Escenario que tiene que ser transformado para la implementación, pues para que los objetivos propuestos sean ejecutados es necesario asegurar y fortalecer la participación ciudadana como complemento y sistema de control del trabajo político de TODOS. Una participación completa, horizontal, diversa y con garantías de respeto y seguridad para todos los actores.

Así como en el acuerdo, legalmente en el país existen varios escenarios concretos que abren la posibilidad de participar y refuerzan la importancia de la acción, el acompañamiento, la apropiación de los colombianos por sus territorios. Desde la Constitución Política Nacional de 1991 se han venido abriendo espacios de participación ciudadana. A nivel territorial, los consejos de ordenamiento territorial, los cabildos abiertos, los Comités Interadministrativos de Educación Ambiental entre otros, son figuras que la comunidad puede aprovechar para ejercer su derecho democrático de participar de las decisiones y del cuidado de su territorio.

Pero hoy nuevamente vemos como la protección a la oposición y a los grupos sociales es precaria y esta carencia, unida a la fuerte presencia de grupos de extrema derecha y el mantenimiento de la mano paramilitar en las regiones sigue cobrando víctimas y sembrando el miedo en el país. Haciendo que las personas no reconozcan estos espacios como lugares seguros o propios de intervención. Entre 2016 y el primer semestre de 2020, han sido masacrados 1008 líderes sociales y defensores de derechos humanos ${ }^{7}$ en todo el país y el gobierno de Iván Duque presenta estos como casos aislados. Incluso en agosto de 2020 el presidente, denomino "Asesinatos Colectivos" a las 34 masacres que se dieron en lo que lleva su mandato, discurso que intenta restarle la gravedad a los terribles hechos que siguen ocurriendo en el país.

\footnotetext{
${ }^{6}$ La mesa de negociación estuvo conformada por una comisión del gobierno y otra de las FARC EP. liderada por el ex-vicepresidente Humberto de La Calle (como jefe negociador) y Sergio Jaramillo Caro (entonces Comisionado de Paz), además de la participación en la mesa de Frank Pearl (excomisionado de paz), Luis Carlos Villegas (empresario colombiano), Enrique Santos (hermano del presidente), Alejandro Éder (consejero presidencial), el general en retiro de la Policía Óscar Naranjo y el también retirado general del Ejército Jorge Enrique Mora, entre otros. En tanto que la delegación de las FARC estuvo integrada por alias Iván Márquez (como jefe negociador), "Jesús Santrich", "Rodrigo Granda", "Simón Trinidad" (recluido en una cárcel de Estados Unidos), "Andrés París", "Marcos Calarcá", "Pablo Catatumbo" (quien ingresó al equipo, meses después del inicio del proceso), Tanja Nijmeijer (insurgente de origen holandés), "Sargento Pascuas", "Mauricio Jaramillo", entre otros.

${ }^{7}$ Información presentada por el Instituto de Estudios para el Desarrollo y la Paz (Indepaz), en su último informe de agosto de 2020. Disponible en: http://www.indepaz.org.co/pazparaliderar/
} 
Como se ve, el panorama no es fácil, y depende de muchos factores. El papel de la sociedad civil es el de garantizar el acompañamiento, control y vigilancia para que sus derechos, los de la tierra y la nación como un todo sean respetados. Para esto es indispensable un proceso de educación con y para la comunidad que permita superar estos miedos y combatir con ideas y acciones concretas las dificultades que alejen de la construcción de una verdadera paz. Ya en Colombia se ha demostrado como la unión de sus gentes ha permitido la superación de tantos años de violencia, de conflicto. En el libro Colombia Compleja Julio Carrizosa Umaña (2014) resalta como en medio de la historia de este país, Colombia se construye con sus gentes y en el descubrir propio del valor de su biodiversidad. Más adelante en su Conferencia: Saberes ambientales y el posconflicto reconoce como esta característica se convierte en una de las claves para construir esa paz que se espera:

Firmada la paz un dialogo de saberes puede conducir al buen vivir en el campo y en la ciudad. En un territorio tan complejo como el colombiano solo la acción conjunta de saberes científicos, personales, colectivos y étnicos puede evitar que recurramos nuevamente a la violencia (CARRIZOSA-UMANA, 2015 p.13).

Garavito et al., (2017) presentan algunos de los desafíos y características de lo que denominan la paz ambiental. Entre lo destacado por los autores, llama la atención el termino democracia ambiental, que ellos definen como: "el derecho que tienen los ciudadanos, las comunidades y las entidades locales de participar en las decisiones relacionadas con el medio ambiente que los afecten" (p.94). Pero aclaran que para que esto sea posible le exige al país una democracia participativa en la que el ciudadano pueda participar de las decisiones públicas. Esto es fundamental pues como lo plantean los autores, las formas en que se ordena y piensa el territorio deben tener en cuenta a quienes viven y sobreviven en los mismos, evitando agravar las tensiones entre ciudadanos y estado, pero sobre todo entendiéndola como la manera de potencializar un desarrollo más equitativo y sostenible.

\section{Como Concretar Estas Ideas en los Territorios}

La participación es un mecanismo que para ser válido en su fuerza necesita de ser incentivado. No seremos libres en países democráticos mientras nuestra voz no se siente en los escenarios o esferas políticas, mientras nuestra libertad nos la roba la búsqueda de condiciones para sobrevivir, porque no tenemos garantía de los mínimos para vivir. Colombianos y brasileños deben más que nunca apropiarse de derechos que se dan en el papel y hacer cumplir y valer su implementación.

Consolidar escenarios de paz y democráticos en Colombia y Brasil, y mucho más, hacer de estos estados, países dueño de sus recursos, 
conocedores de su territorio, amantes de la vida que florece en todas las esquinas, en condiciones de un Buen Vivir para todos, es un sueño que solo se materializará con la participación de todos y cada uno de sus habitantes. Cuando el objetivo común es la vida en comunidad, es el cuidar, el proteger desde la igualdad de seres y saberes, es posible superar las diferencias y hacer transformaciones significativas. El conocer nuestro territorio, ser conscientes de nuestra responsabilidad y nuestro papel en el desarrollo de este, en el reconocimiento de que somos parte y que hacemos parte de nuestro ambiente, nos hace percibir de una manera más compleja las relaciones que se presentan en el territorio y por lo tanto empezar a actuar diferente:

El territorio es construido simbólicamente. Quienes lo habitan elaboran permanentemente representaciones sobre su vida en el territorio tales como modos de vivir y de concebir la existencia individual y colectiva; ligándose a él a través de la construcción de sentidos de pertenencia, marcados culturalmente y de intensidad afectiva diferente según la escala territorial de referencia. (...) el territorio es construido políticamente a través de la toma de decisiones sobre el destino que sus habitantes prefiguran para él. Tales decisiones revelan las asimetrías existentes entre los diferentes grupos sociales, en términos de los recursos de poder que cada uno de ellos tiene a su alcance para imponer su propio proyecto territorial (VELÁSQUEZ, 2012, p.244).

Espacios que, como ya se ha dicho en este texto, han sido vulnerados o poco aprovechados por la comunidad, pero que, desde su reafirmación, serán la herramienta clave para que el ciudadano pueda luchar por el cumplimiento de estos y otros derechos primero en su territorio y con ello en el país. La reconstrucción del territorio es esencial para la construcción de una Colombia y de un Brasil diferentes. Al garantizar que sean respetaos los derechos sociales y el acceso a la tierra, el cuidado y la conservación de las tierras, animales, plantas, recursos minerales, agua y las poblaciones, no solo se comienza el fin del conflicto, como se garantiza el crecimiento social y cultural de los países. Esto implica, como lo propone Ángel (2015):

Ordenar ambientalmente el territorio a partir del respeto y el reconocimiento de la estructura ecológica del mismo y de las diferentes territorialidades presentes en él. Se requiere entonces redefinir la relación campo-ciudad, fortalecer el Sistema Nacional Ambiental y profundizar en su gobernanza. (p.90).

Generar una revolución educativa que, gracias a la producción de conocimiento, permita la comprensión integral del país en que vivimos, un entendimiento detallado de las redes vitales y sociales que sostienen nuestro territorio, con miras a propiciar una nueva ética ambiental y a fortalecer nuestros niveles de 
resistencia (p.91).

Esta es la importancia de tener al territorio como centro del debate en cuanto a construcción de paz y de buen vivir. Las poblaciones empoderadas de sus territorios y con estas posibilidades de protección y participación, podrán ser actores críticos de los cambios necesarios para potencializar y mejorar las condiciones de vida para todos y todas según sus particularidades. Es necesario cambios radicales en la forma de entender la importancia de nuestro papel en la construcción de nuestros países, el miedo y la apatía no pueden ser más los ejes de la vida de la población. Y aunque estamos muy lejos de garantizar que estas condiciones sean ofrecidas, si es necesario y urgente empezar a dialogar en cada comunidad, para conocernos para identificar nuestras características, particularidades, naturales, sociales, culturales, históricas y desde allí continuar.

En la política de EA de Brasil (1999) y Colombia (2002), la participación tiene una función muy importante en el discurso, pues se le coloca a la organización social la responsabilidad de la implementación de lo que allí se propone. Estas propuestas incentivan a que sean los mismos actores, en cada contexto, los encargados de proponer y poner en práctica estrategias que se orienten al cumplimiento de lo estipulado en la política, pero desde el reconocimiento del contexto, de las necesidades y potencialidades de cada territorio. Estas políticas serían un referente teórico y de gestión que pueden articular las iniciativas regionales. La EA propuesta por los dos países plantea además un diálogo de saberes, que involucra las diferentes culturas y saberes, así como diferentes formas institucionales. Lo que implican desde el comienzo un gran desafío para la política de EA, pues la participación, la concertación y la gestión necesitan de condiciones para su verdadera aparición en los territorios.

Las garantías deben incluir desde recursos físicos y técnicos, hasta condiciones de diálogo e igualdad que permita la libertad de los actores, y para que todos los saberes tengan el mismo lugar en la mesa de conversación. A pesar de que en las dos políticas la participación es propuesta insistentemente no se identifican estrategias concretas para comenzar a integrar saberes otros al discurso (MEJIA TORO, 2018). Las acciones de integración y acompañamiento propuestas en las políticas e implementados por los Programas de EA en los dos países han enfocado sus esfuerzos a la organización de la sociedad en torno a espacios e instituciones formales.

La figura del órgano gestor de la EA para el Brasil, así como el programa de EA en Colombia, son producto del discurso que defiende la fuerza de la participación. Los dos sistemas hacen una invitación a todos los sectores sociales: a los diferentes niveles de gobierno, empresarial, sociedad civil, ONGS, representantes de minorías, etc. a participar de la toma de decisiones ambientales o de EA. No todos ellos tienen una participación efectiva en estos escenarios, dejando ver una dificultad en la implementación real de escenarios 
de participación de este tipo. Pues, aunque las estructuras organizativas están descritas ampliamente en las políticas de EA de cada país e incluso documentos específicos, en la práctica no se ha dado una continuidad a la participación de la mayoría de estos actores.

En ambos países se ha avanzado en las estrategias de participación local los Proyectos Ambientales Escolares PRAES, los Comités Técnicos Interinstitucionales de Educación Ambiental CIDEA, en Colombia; y las salas verdes, las conferencias ambientales, los Colectivos Educadores entre otros en Brasil. Estos espacios proponen ir agregando personas a la propuesta nacional y construir escenarios para pensar y hacer EA en el territorio. Espacios que son un mecanismo para iniciar procesos de articulación entre los grupos sociales y los discursos de EA en cada país, pero aún es necesario trabajar en la efectividad de esta participación con el objetivo de garantizar que en los territorios todos sean llamados y puedan convocar discusiones educativo-ambientales.

Congresos Iberoamericanos de EA, así como foros y otros eventos que han ocurrido en diversos países de la región, en las últimas décadas, han promovido relatos de experiencias, diálogos sobre ideas y propuestas que incentivan revisitar y crear/revitalizar sueños y utopías colectivas, como también la determinación por realizarlos. Algunas directrices que pueden ser interpretadas de la lectura de esos relatos y pueden servir como subsidios para la formulación e implementación de estrategias de participación efectiva desde el fortalecimiento de la EA son: articulación de los actores locales para formular e implantar un proyecto político-pedagógico territorial que incentive la formación de círculos de aprendizaje participativa que ejercitan la auto gestión y el auto análisis como práctica dialógica cotidiana, emuladora de valores comprometidos con la sustentabilidad socio ambiental; dar testimonio de los valores promulgados en el interior de cada institución y de personas participantes así como de la relación entre ellas; aproximación y compromiso entre las fuerzas instituidas y las instituyentes que actúan en cada territorio; realización de diagnósticos, monitoreo y evaluación continuada del estado de arte del área, por medio de Observatorios de EA conectados en Red, entre sí y con otros volcados al acompañamiento de cuestiones sociales y ambientales; implantación por el estado, en sus diversas esferas e instancias, de estructuras de apoyo (recursos humanos, financieros, políticos y materiales) a las articulaciones colectivas y territoriales de EA; promoción al acceso a las informaciones, reflexiones y acciones colectivas orientadas a enfrentar cada uno de los desafíos apuntados en el ítem anterior; apoyo a las iniciativas de fondos públicos, no estatales, donde el estado sea uno de sus actores, con el objetivo de imprimir agilidad y control social en la destinación y utilización de recursos para una EA pautada por instancias de formulación de políticas públicas en el área; fortalecimiento de los programas de Educación ambiental nacionales que articulen, de forma orgánica, todas las iniciativas de EA, gubernamentales y no gubernamentales, en cada país. Articulación entre los programas de EA nacionales con el objetivo de ampliar la incidencia de la EA 
latinoamericana en el panorama internacional; definición de los territorios de los Municipios, como prioridad para la EA, promoviendo la aproximación entre Instituciones de Educación Superior - IES, Escuelas, Administración Municipal y las demás instituciones y movimientos constituyentes de la EA, para juntas enfrentar los desafíos del área.

Aprovechar los espacios ya creados y respaldaos por ley, en una efectiva implementación; así como la generación de nuevos espacios populares y comunitarios, puede provocar diálogos entre actores del conflicto, entre pares, entre pueblo y estado para la reconstrucción y establecimiento de territorios de paz. La paz no se limita al cese al fuego y de hostilidades. La paz de un país implica una democracia justa y participativa en el que sean respetados los derechos y cumplidos los deberes, donde se respete el territorio, la diversidad biológica y cultural. Esto es emprender un camino en el que estos sean realmente los valores respetados y cumplidos por todos, garantizará verdaderos cambios que conduzcan a la paz. Un nuevo paradigma en el que desde el gobierno se generen políticas sociales y condiciones de vida, cuidado y sobrevivencia y en el que los ciudadanos sepan y puedan defender su territorio, sus derechos y sean partícipes activos de estas transformaciones.

Esto solo es posible desde una comprensión de nuestro pasado, un empoderamiento de nuestro presente y las perspectivas de futuro común, en el que la participación y el empoderamiento de los pueblos será la garantía de transformación.

\section{Consideraciones Finales}

Brasil y Colombia son dos países que comparten sus más grandes orgullos y al mismo tiempo sus más grandes pesares. Siendo los países líderes en biodiversidad, en riqueza natural y en diversidad cultural, presentan también conflictos internos en los que la violencia pone en jaque la viabilidad de estos sistemas. La histórica violencia que ha manchado sus territorios ha generado estructuras de poder en los que la democracia se ha visto gravemente afectada, pues el miedo y la violencia han debilitado las formas de participación política y social de sus comunidades. Y es así como hoy en medio de la violencia política, social y natural que se viven en estos países es más que nunca fundamental la organización de la población para defender su derecho a la libertad, a la democracia, al ambiente y a la vida digna.

Uno de los principales mecanismos para esta transformación cultural es la educación, una educación que desde una perspectiva ambiental tiene el potencial de promover la participación con todos y cada uno desde el reconocimiento y empoderamiento del territorio. Una EA que, aunque ya propuesta desde la ley, no ha acompañado efectivamente el desarrollo de estos procesos. Es necesario valorar y reforzar estas estrategias y metodologías, para que penetren los escenarios territoriales y se conviertan efectivamente en motores de transformación socio ambiental.

revista brasileira educação ambiental 
La educación ambiental tiene el potencial de convertirse en un hilo conductor que permitiría el empoderamiento de las comunidades a partir del auto reconocimiento de sus territorios, su diversidad, su riqueza ambiental. La educación ambiental, por los ideales que plantea, se ofrece como uno de los tantos espacios que permiten el encuentro y el dialogo de todos los actores de la comunidad y con ello se permita la posibilidad de construcción de paz. La educación pensada desde lo ambiental no es diferente a la educación para la paz, las dos comparten los mismos objetivos. La educación de corte ambiental parte desde el reconocimiento de nuestras características socioambientales para desde allí construir comunidad, territorio, país.

\section{Nota:}

En el periodo en que se edita y publica este texto, Colombia experimenta una movilización social sin precedentes para el país. Un movimiento que se reúne en las calles para demandar del gobierno, entre otras cuestiones, justicia social, la implementación de los acuerdos de paz y justicia por la muerte de líderes y lideresas sociales. Las manifestaciones comenzaron en 2019, pero fueron interrumpidas por la pandemia SARS-CoV 2 - Covid 19. Ya en 2021, la crisis económica y social, un paquete de reformas tributaria, laboral y pensional, enardecieron los ánimos y llevaron al pueblo de nuevo a las calles.

Aplaudimos que son la juventud y los grupos étnicos quienes lideran la protesta social por medio de un paro que ha usado la pedagogía y el arte para hacer sentir sus inconformidades. A su vez repudiamos y denunciamos la respuesta del gobierno que ha sido indolente y ha usado contra los manifestantes la fuerza pública y un discurso que violenta el derecho a la movilización. Un ejemplo son las palabras de la canciller y vicepresidenta, quien culpa a el proceso de paz, específicamente al punto 2, por promover un país en "modo paro" como algo negativo pues generaría la falta de "paz social". Esto ha provocado escenarios de extrema violencia con numerosos heridos, desaparecidos y muertos. Solo en la ciudad de Calí, el Informe Preliminar № 1 del 28 de mayo de 2021 hecho por la organización Comisión Por La Vida ${ }^{8}$, reporta 46 asesinatos, 93 desapariciones y 240 detenciones. Mientras que organizaciones como el Instituto de estudios para el desarrollo y la paz Indepaz ${ }^{9}$ reportan 71 víctimas de violencia homicida en el territorio nacional. Violencia ejercida principalmente por civiles armados y las fuerzas armadas del estado, principalmente el Escuadrón Móvil Antidisturbios ESMAD.

\section{Referências}

ALVINO, S.; SESSANO, P. La Educación Ambiental como herramienta para el

\footnotetext{
${ }^{8}$ Comisión conformada por: Red Amplia La Colcha, Unión de Ciudadanas, Ruta Pacífica, movimiento Antiracista, Afrodesc, Nomadesc, Vicaría para el Servicio, Observatorio de Realidades Sociales de la Arquidiócesis, Faculta de Educación de Unicatólica, Instituto de Paz Univalle, Red de Hermandad y Solidaridad con Colombia, Integrados y Ciudad en Movimiento. https://observatoriorealidades.arquidiocesiscali.org/wp-content/uploads/2021/05/Que-cese-lahorrible-noche-informe-violancion-D.H.-Comisio\%CC\%81n-Por-La-Vida.pdf

${ }^{9}$ http://www.indepaz.org.co/victimas-de-violencia-homicida-en-el-marco-del-paro-nacional/
}

Revbea, São Paulo, V. 16, № 4: 320-339, 2021. 
ordenamiento territorial: una experiencia de política públicaDiez años de cambios en el Mundo, en la Geografía y en las Ciencias Sociales, 1999-2008: Actas del X Coloquio Internacional de Geocrítica. Barcelona. Disponível em: http://www.ub.edu/geocrit/-xcol/programa.htm.

ÁNGEL, M. Conferencia magistral Una mirada desde la educación ambiental al posconflicto colombiano. In: FORO NACIONAL EDUCACIÓN AMBIENTAL Y POSCONFLICTO AMBIENTE PARA LA PAZ. MEMORIAS. MINISTERIO DE AMBIENTE $Y$ DESARROLLO SOSTENIBLE Y ALCALDÍA MAYOR DE BOGOTÁ. 2015a, Bogotá D.C. Anais [...]. Bogotá D.C.

ÁNGEL, M. Conferencia magistral Una mirada desde la educación ambiental al posconflicto colombiano. In: FORO NACIONAL EDUCACIÓN AMBIENTAL Y POSCONFLICTO AMBIENTE PARA LA PAZ. MEMORIAS. MINISTERIO DE AMBIENTE $Y$ DESARROLLO SOSTENIBLE Y ALCALDÍA MAYOR DE BOGOTÁ. 2015b, Bogotá D.C. Anais [...]. Bogotá D.C.

ARISTIZÁBAL, A.M.D. Educación ambiental: Una mirada desde Colombia. Tópicos en educación ambiental, [S. I.], v. 1, n. 3, p. 7-16, 1999.

BRAGA SOUSA, M.S.; ACUÑA CHAVERRI, I.A. El fortalecimiento de la democracia en Brasil y los retos de la observación electoral. América Latina Hoy, [S. I.], v. 70, p. 91, 2015.

CARRIZOSA-UMAÑA, J. Conferencia Magistral: Los saberes ambientales en el postconflicto. Segundo Congreso de Ciencias y Tecnologías Ambientales de la Red de Formación Ambiental. Memorias Congreso Nacional de Ciencias Ambientales. In: 2015, Pereira - Risaralda. Anais [...]. Pereira - Risaralda Disponível http://www.congresocienciasambientales.com/memorias/Conferencias

Magistrales/Dr. Julio Carrizosa Umaña. Los saberes ambientales en el posconflicto.pdf.

CARRIZOSA UMAÑA, J. Colombia compleja. [s.I: s.n.]. Disponível em: http://hdl.handle.net/20.500.11761/32548.

CHESNAIS, J.C. A Violência no Brasil . Causas e recomendações políticas para a sua prevenção Violence in Brazil : causes and politic recommendations to its prevention. Ciência \& Saúde Coletiva, [S. I.], v. 4, n. 1, p. 53-69, 1999.

FERRARO, L.A.; SORRENTINO, M. Imaginário Político e Colonialidade: Desafios à Avaliação Qualitativa das Políticas Públicas de Educação Ambiental. Ciência \& Educação, [S. I.], v. 17, n. 2, p. 339-352, 2011.

FRANCO, J.L.A.; DRUMMOND, J.A. Proteção à natureza e identidade nacional no Brasil, anos 1920-1940. In: [s.l.] : Editora FIOCRUZ., 2009.

FRANCO, J.L.A.; DRUMMOND, J.A. História das preocupações com o mundo natural no Brasil: da proteção à natureza à conservação da biodiversidade. In: História ambiental: fronteiras, recursos naturais e conservação da natureza. [s.I.] : Editora Garamond Ltda., 2012. 
FREIRE, P. Pedagogia del Oprimido. 1970.

GARAVITO, C.R.; FRANCO, D.R.; CRANE, H.D. La paz ambiental: retos y propuestas para el posacuerdo. In: JUSTICIA, De (org.). Documentos 30: Ideas para construir la paz. [s.l: s.n.]. Disponível em: https://www.dejusticia.org/wp-

content/uploads/2017/04/fi_name_recurso_924.pdf.

INSTITUTO DE PESQUISA ECONÔMICA APLICADA; FÓRUM BRASILEIRO DE SEGURANÇA PÚBLICA. Atlas da Violência. [s.l: s.n.]. Disponível em: https://ipea.gov.br/atlasviolencia/.

LUCA, A.Q. Uma análise de discurso da política pública federal de educação ambiental. 2013. Tese de Doutorado, Universidade de São Paulo, São Paulo, 2013. DOI: 10.11606/T.90.2013.tde-04112013-104411. Disponível em: https://www.teses.usp.br/teses/disponiveis/90/90131/tde-04112013-

104411/publico/andrea_quirino_luca_tesecorrigida.pdf.

MEJIA TORO, L.F. Estudio comparado de las políticas públicas de educación ambiental de Brasil y Colombia. 2018. Universidade Federal de Minas Gerais, [S. I.], 2018.

MORALES, L. La paz y la protección ambiental en Colombia: propuestas para un desarrollo rural sostenible. [s.l: s.n.]. Disponível em: http://static.iris.net.co/sostenibilidad/upload/documents/envt-colombia-esp_webres_final-for-email.pdf.

PRESIDENCIA DE LA REPUBLICA; FARC-EP. Acuerdo Final para la Terminación del Conflicto y la Construcción de una Paz Estable y Duradera. [s.l: $\quad$ s.n.]. Disponível em: http://www.centrodememoriahistorica.gov.co/descargas/finAcuerdoPazAgosto2 016/12-11-2016-Nuevo-Acuerdo-Final.pdf.

SCHITTINI, G.; FRANCO, J.; DRUMMOND, J. Expansão da fronteira de recursos naturais e unidades de conservação da natureza na área de influência da BR - 163. In: RIO DE JANEIRO: GRAMOND (org.). História ambiental: Fronteiras, recursos naturais e conservação da natureza. [s.l: s.n.]. p. 392.

SORRENTINO, M.; RAYMUNDO, M.; PORTUGAL, S.; RAYMUNDO, A.; MORAES, F.C.; SILVA, R.F. Educação, Agroecologia e Bem Viver: transição ambientalista para sociedades sustentáveis. [s.l: s.n.]. Disponível em: http://www.nacepteca.esalq.usp.br/sites/default/files/publicacao_arq/Educacaoagroecologia-e-bem-viver final.pdf.

SOTERO, J.P. O Financiamento Público da Política Nacional de Educação Ambiental: do Veto Do Artigo 18 às Novas Estratégias de Financiamento. 2008. UNIVERSIDADE DE BRASÍLIA, [S. I.], 2008. Disponível em: http://www.reasul.org.br/files/dissertacao_Sotero.pdf. 
capitais The panorama of urban violence in Brazil and its Capitals. Ciência \& Saúde Coletiva, [S. I.], v. 11, p. 1211-1222, 2007.

VELÁSQUEZ, F. Participación y Ordenamiento Territorial en Colombia. In: Ordenamiento Territorial y Participación. Dialogo Territoria. Grupo Gadi ed. Bogotá: Instituto Geográfico Agustín Codazzi., 2012. p. 243-258.

WELLE, D. Notícias Brasil, Meio Ambiente. [S. I.], 2020. Disponível em: https://www.dw.com/pt-br/brasil-é-o-terceiro-país-mais-letal-do-mundo-paraativistas-ambientais/a-54361835. 